\title{
Effect of Histone Deacetylase Inhibitor to Osteosarcoma through Specificity Protein 1/ Phospholipase D1 Pathway
}

\author{
DAWEI. CAI ${ }^{1}$, TANGBO. YUAN ${ }^{1}$, ZHEN JIN AND JIAN QIN*1
}

Department of Orthopaedics, The Second Affiliated Hospital of Nanjing Medical University, Nanjing, Jiangsu 210011, ${ }^{1}$ Department of Orthopaedics, Sir Run Run Hospital, Nanjing Medical University, Nanjing, Jiangsu 211000, P.R. China

Cai et al.: Effect of Histone Deacetylase Inhibitor to Osteosarcoma

To study the effect of histone deacetylase inhibitor to osteosarcoma by targeting specificity protein 1 and regulating Phospholipase D1. Human osteosarcoma cell line MG63 cells were cultured in Dulbecco's Modified Eagle's medium medium containing penicillin $(100 \mathrm{U} / \mathrm{ml})$ and $10 \%$ fetal bovine serum at $37^{\circ}$. siRNA-specificity protein 1 and psiCHECK-Phospholipase-WT plasmid were constructed and transfected into MG63 cells. Dual luciferase reporter gene assay were conducted to verified the combination site of specificity protein 1 and Phospholipase. MG63 cells were treated with tumor-specific antigen (5 g/ml) or PBS (negative control) for $48 \mathrm{~h}$ at $37^{\circ}$. Western blot was used to test the phospholipase D1, nuclear specificity protein 1 and cytoplasmic specificity protein 1 in MG63 cells. Cell Counting Kit-8 and clone formation assay were conducted to analyze the difference in cell viability and proliferation of tumorspecific antigen -treated MG63 cells and tumor-specific antigen free cells. Western blot result showed that nuclear and cytoplasmic specificity protein 1 in tumor-specific antigen treated MG63 cells both decreased dramatically compared to tumor-specific antigen free cells $(\mathbf{p}<\mathbf{0 . 0 5})$. Phospholipase in tumor-specific antigen treated MG63 cells also reduced $(\mathbf{p}<\mathbf{0 . 0 5})$. Fluorescence analysis showed that the ratio of Firefly luciferase to Renilla luciferase in MG63 cells transfected with psiCHECK-Phospholipase-WT plasmid and si-specificity protein 1 was significantly lower than that of si-NC $(p<0.05)$, while the ratio of firefly luciferase to Renilla luciferase in MG63 cells transfected with psiCHECK-Phospholipase-WT plasmid and si-specificity protein 1 had no significant difference with si-NC control $(p<0.05)$. After tumor-specific antigen treatment for $24 \mathrm{~h}$, the proliferation rate of MG63 cells was (65.20\% $\pm 2.31 \%)$, which was significantly lower than that of PBS control group $(96.63 \% \pm 1.14 \%)(p<0.05)$ by CCK-8 method. Cell formation assay showed that the amount of MG63 cells treated with tumor-specific antigen decreased significantly $(p<0.05)$. The gene expression of specificity protein 1 and Phospholipase in tumor-specific antigen -treated MG63 cells decreased significantly compared with tumor-specific antigen free cells $(p<0.05)$. The expression of survivin in tumor-specific antigen -treated MG63 cells were significantly lower than that in tumor-specific antigen free cells $(\mathbf{p}<0.05)$. The gene expression levels of p21 in tumor-specific antigen -treated MG63 cells were significantly higher than that in tumor-specific antigen free cells $(p<0.05)$. The expression of cyclin A2 had no significant difference between two groups $(\mathbf{p}<\mathbf{0 . 0 5})$. Tumor-specific antigen can increase the early apoptosis rate, late apoptosis rate and total apoptosis rate of MG63 cells compared to the non- tumorspecific antigen -treatment cells $(\mathbf{p}<\mathbf{0 . 0 5})$. Specificity protein 1 could bind and target with Phospholipase promotor/enhancer region. Histone deacetylase inhibitor can reduce the proliferation and apoptosis of osteosarcoma cells through decreasing the expression of Phospholipase by inhibiting the protein expression and nuclear migration of specificity protein 1).

Key words: Osteosarcoma, histone deacetylase inhibitor, trichostatin A, specific protein 1, phospholipase D1

Osteosarcoma is a common and highly malignant bonerelated tumor. It occurs frequently in 10 to $30 \mathrm{y}$ old adolescents, ranking number two in cancer-related deaths in children and adolescents ${ }^{[1]}$. In recent years, increasing new drugs have been used to treat osteosarcoma,but its

*Address for correspondence

E-mail: jian_qin_njmu@sina.com

November-December 2020
This is an open access article distributed under the terms of the Creative Commons Attribution-NonCommercial-ShareAlike 3.0 License, which allows others to remix, tweak, and build upon the work non-commercially, as long as the author is credited and the new creations are licensed under the identical terms

Accepted 24 December 2020

Revised 15 October 2020 Received 20 July 2020 Indian J Pharm Sci 2020;82(6):1025-1031 
prognosis is still less than satisfactory ${ }^{[2]}$, the $5 \mathrm{y}$ survival rate of osteosarcoma is only 50-60\%. Poor prognosis is associate to complex cell microenvironment of osteosarcoma which involves multiple factors and multiple pathways. Common chemotherapy lack sensitive targets for tumor cell recognition, and can easily lead to drug resistance. Therefore, osteosarcoma has its special pathogenesis and special target unlike other cancer. The treatment strategy based on traditional target and single target become more and more limited. Finding new targets to improve the efficacy of drug therapy, or developing new clinical drugs to achieve multiple targets of one drug are still the hopeful research directions for clinical breakthrough.

Human nuclear transcription factor specific protein 1 (SP1), a member of SP/KLF family, is widely expressed in vivo. It can specifically recognize $\mathrm{GC}$ rich elements in deoxyribonucleic acid (DNA) and participate in the transcriptional regulation of many genes. At present, SP1 is widely accepted as a tumor promoting protein. The expression of SP1 protein is low or absent in normal tissues, but high in gastric cancer, pancreatic cancer, breast cancer and thyroid tumor cells ${ }^{[3]}$. Phospholipase D1(PLD1) is an important enzyme that regulates the metabolism of phospholipids. It catalyzes the hydrolysis of phosphatidylcholine to produce phosphonic acid (PA). PA plays an important role in cancer-related signal pathway. Most of its downstream target proteins have been confirmed to be related to cell proliferation and metastasis ${ }^{[4]}$. Both SP1 and PLD1 are independent tumor risk factors and interact with each other ${ }^{[5]}$. It is still not clear how SP1 and PLD1 regulate in osteosarcoma cells. In our previous study, we compared the tumor samples of 137 patients with osteosarcoma and found that the contents of SP1 and PLD1 in osteosarcoma tissues were significantly higher than those in adjacent tissues. The follow-up results showed that the increased expression of the two proteins in osteosarcoma tissue could lead to poor prognosis of patients with osteosarcoma ${ }^{[6]}$, SP1 and PLD1 may be potential therapeutic targets for osteosarcoma.

Histone deacetylase inhibitor (HDACi) is a new kind of antitumor drugs, which can block cell cycle, induce cancer cells apoptosis and differentiation. In 2006, the Food and Drug Administration (FDA) officially approved the first HDACi, Zolinza (vorinostat) to treat cutaneous T-cell lymphoma. At present, Zolinza has developed to treat a variety of hematological and solid tumors ${ }^{[7,8]}$. In order to further study the effet and the mechanism of HDACi for osteosarcoma, we choose a typical HDACi trichostatin A (TSA) to treat osteosarcoma cells in vitro, and intend to demonstrate if TSA can antitumor by targeting SP1 and PLD, and clarify the effect of SP1 after intervention of TSA. We intend to explore the specific mechanism of combined action of SP1 and PLD1 in tumor progression process, and provide a new target and a new drug for clinical treatment of osteosarcoma, and a new idea for clinical reversal of chemotherapy resistance of osteosarcoma.

\section{MATEIRALS AND METHODS}

\section{Materials:}

Human osteosarcoma cell line MG63 (ATCC, CAT. NO. CRL-1427); penicillin, fetal bovine serum (FBS) (Sigma-Aldrich, USA), Lipofectamine $2000^{\mathrm{TM}}$ (Invitrogen, CAT. NO. 11668-027), PLD1 rabbit polyclonal antibody (Absin, Shanghai, CAT. NO. ABS137279), SP1 rabbit polyclonal antibody (AtaGenix CAT. NO. ATA37845), nuclear and cytoplasmic protein extraction kit (Beyotime, CAT. NO. P0027), Cell Counting Kit (CCK-8, DOJINDO, CK04), VaripsKan Flash Microplate Reader (Thermo Scientific, USA), Trizol reagent (Invitrogen, CAT. NO. 15596-026).

\section{Treatment of MG63 cells with TSA:}

MG63 cells were resuscitated and cultured in Dulbecco's Modified Eagle's medium (DMEM) medium containing penicillin $(100 \mathrm{U} / \mathrm{mL})$ and $10 \% \mathrm{FBS}$ at $37^{\circ}$ and $5 \%$ $\mathrm{CO}_{2}$. When MG63 cells overspreaded more than 80 $\%$ of the culture dish, trypsin was added for digestion and cell suspension was washed by PBS solution and collected by centrifugation. MG63 cells were diluted by DMEM medium into $2 \times 10^{5}$ cells and transfered into 96 well plate at $37^{\circ}$ and $5 \% \mathrm{CO}_{2}$ for $24 \mathrm{~h}$. Then MG63 cells were treated with TSA $(5 \mathrm{~g} / \mathrm{ml})$ or PBS (negative control) for $48 \mathrm{~h}$ at $37^{\circ}$ in a $5 \% \mathrm{CO}_{2}$, six parallel wells for each treatment. After treatment with TSA, all medium was discarded and cells were harvested for following experiments.

\section{Separation of nuclear and cytoplasmic protein:}

After cell collection, we performed cytoplasmic and nuclear fractions separation by using nuclear and cytoplasmic protein extraction kit. In brief, cells treated as indication were resuspended in PBS, followed by addition to cytoplasmic protein extraction reagent $\mathrm{A}$ and $\mathrm{B}$. Then, the samples were centrifuged, and the supernatant was collected to obtain cytoplasmic fraction. The nuclei was resuspended in nuclear protein 
extraction agent. After centrifugation, the supernatant was collected as the nuclear fraction.

\section{Western blot:}

Nuclear and cytoplasmic protein was respectively treated by radioimmunoassay (RIPA) buffer and determined concentration by BCA method. The protein was heated at $95^{\circ}$ for 5 min to lysate and was dissolved in $30 \mathrm{~min}$ by ice bath. Then the protein was extracted by dodecyl sulfonate polyacrylamide gel electrophoresis (SDS-PAGE) and transferred to the polyvinylidene fluoride (PVDF) membrane. The PVDF membrane was sealed by $5 \%$ skimmed milk powder at room temperature and the added 1:1000 first antibody of SP1, PLD1 and $\beta$-actin at room temperature overnight. After washing with TBST, the PVDF membrane was added into HRP-labeled second antibody (diluted with 1:5000). After incubation for $1 \mathrm{~h}$ at room temperature, the membrane was rinsed with TBST for three times, the positive bands were displayed with chemiluminescent reagent, and the images were processed and analyzed with gray-scale analysis software.

\section{Down-regulation of SP1 in MG63 cells:}

MG63 cells were resuscitated and cultured in DMEM medium containing penicillin $(100 \mathrm{U} / \mathrm{mL})$ and $10 \% \mathrm{FBS}$ at $37^{\circ}$ and $5 \% \mathrm{CO}_{2}$. When MG63 cells overspreaded more than $80 \%$ of the culture dish, trypsin was added for digestion and cell suspension was washed by PBS solution and collected by centrifugation. To down-regulation of SP1, si-SP1 was synthesized by Shanghai JIKAI GENE Chemistry Technology Co., Ltd. To synthesize siRNA-SP1, the forward primer was 5'-ATTCGATCGGGGCGGGGCGAGC-3' and reverse primer was 5'-AUAGUUGAGUCATGT-3'. siR-SP1 was transfected into MG63 cells by Lipofectamine $2000^{\mathrm{TM}}$.

\section{Dual luciferase reporter gene assay:}

According to the sequence of PLD1 promoter/enhancer region (-154 to $-109,45 \mathrm{bp})$, two reverse complementary DNA primers were designed. The primers were heated and then linked with dual luciferase reporter to construct psiCHECK-PLD1-WT plasmid. psiCHECK-PLD1-MT were constructed as mutation of psiCHECK-PLD1WT for negative control. The construction process was completed by Shanghai Jima Company.

MG63 cells after transfection with si-SP1 or si-NC were harvested and adjusted the cell density to $1 \times 10^{5}$ cells $/ \mathrm{ml}$. the cells were transferred on a 96 well plate with $0.1 \mathrm{ml}$ per well. It was incubated overnight in a $5 \% \mathrm{CO}_{2}$ incubator at $37^{\circ}$. The psiCHECK-PLD1-WT and psiCHECK-PLD1-MT plasmid were respectively transfected into MG63 cells by Lipofectamine $2000^{\mathrm{TM}}$. After transfection, the culture medium was discarded and washed with $100 \mu \mathrm{l}$ PBS for two times; PLB solution was added to the cells at room temperature for $50 \mathrm{rpm} / \mathrm{min}$ for $15 \mathrm{~min}$. The cell lysate was collected; $20 \mu \mathrm{L}$ cell lysate and $100 \mu \mathrm{LAR}$ II were added to each well of the black opaque 96 well enzyme label plate, and the data were detected automatically after standing in dark for $2 \mathrm{~s} ; 100 \mu \mathrm{l}$ STOP \& GLO Regent was added into each well, and the data was detected automatically after standing in dark for $2 \mathrm{~s}$.

\section{Cell proliferation analysis:}

Cell Counting Kit was used to measure the effects of TSA on cell viability ${ }^{[9]}$. Briefly, After indicated TSA exposure, $10 \mu 1$ of CCK8 reagent was added followed by incubation for another $2 \mathrm{~h}$ at $37^{\circ}$ and $5 \% \mathrm{CO}_{2}$. The optical density (OD) value at $450 \mathrm{~nm}$ was recorded by the Microplate Reader.

\section{Clone formation assay:}

$1.2 \%$ and $0.6 \%$ low melting point agarose solutions were sterilized and ketp in semisolid state by $40^{\circ}$ water bath. $1.2 \%$ low melting point agarose solution and $2 \times$ DMEM medium (containing 20\% FPS) were mixed and transfered into 6 well plate as the bottom agar. $0.3 \%$ soft agar was prepared by mixing $0.6 \%$ low melting point agarose solution and $2 \times$ DMEM medium (containing $20 \%$ FPS).

The MG63 cells treated with TSA or PBS were harvested by centrifuging at $1500 \mathrm{rpm}$ for $10 \mathrm{~min}$. The supernatant was removed and washed twice with PBS solution. The cell density was adjusted with DMEM medium to 500 cells $/ \mathrm{ml} .1 \mathrm{ml}$ of cells solution and $1 \mathrm{ml} 0.3 \%$ soft agar were inoculated into six well plate. Culture in incubator at $37^{\circ}$ and $5 \% \mathrm{CO}_{2}-95 \%$ air mixture for $10 \mathrm{~d}$. The cells were washed with PBS twice, and fixed with methanol for $10 \mathrm{~min}$. The cells were dyed with $0.1 \%$ crystal violet for 20 min and washed with PBS. Under $400 \times$ microscope, the cells were observed in 5 fields randomly and counted the number of colonies.

\section{Quantitative real-time polymerase chain reaction (qRT-PCR):}

Total RNA was isolated with Trizol following the manufacturer's protocol. Total RNAs were reverse transcripted into cDNA using the Reverse Transcription 
kit. qRT-PCR analysis was performed in SYBR GREEN PCR Master Mix in $20 \mu 1$ reactions. The related mRNA expression levels were normalized to the $\beta$-actin by $2^{-\Delta \Delta C T}$. The primers are synthesized by Shanghai Sangon Company ,the primer sequence were listed in Table 1. PCR program was $95^{\circ}: 30 \mathrm{~s},\left(95^{\circ}: 5 \mathrm{~s}, 60^{\circ}: 34 \mathrm{~s}\right) \times 40$ cycles.

\section{Flow cytometry:}

The MG63 cells treated with TSA or PBS were harvested by centrifuging at $1500 \mathrm{rpm}$ for $10 \mathrm{~min}$. The supernatant was removed and washed twice with PBS solution. The cell density was adjusted to $2 \times 10^{6}$ cells/group. Then the MG63 cells were centrifugated to remove the supernatant and replace with pre-cooled $70 \%$ ethanol to incubate at $-20^{\circ}$ overnight. The MG63 cells were centrifugatd at $1500 \mathrm{rpm}$ for $5 \mathrm{~min}$ for removal the supernatant, then washed with PBS twice. Propidium iodide (PI) solution was added into the cells and dyed at room temperature and avoided light for 30 min. Flow cytometry was performed to measure the cell cycle within $1 \mathrm{~h}$.

\section{Statistical analysis:}

SPSS 22.0 software was used for statistical analysis. Data were present as $\left(\mathrm{x}^{ \pm} \mathrm{s}\right)$. Student's T-test was used for comparison between two groups, $\mathrm{p}<0.05$ means there was statistical significance between different groups.

\section{RESULTS AND DISCUSSION}

Nuclear and cytoplasmic SP1 in TSA treated MG63 cells both decreased dramatically compared to TSA free cells $(p<0.05)$. PLD1 in TSA treated MG63 cells also reduced $(p<0.05)$, as shown in fig. 1 .

As shown in fig. 2, fluorescence analysis showed that the ratio of Firefly luciferase to Renilla luciferase in MG63 cells transfected with psiCHECK-PLD1-WT plasmid and si-SP1 was significantly lower than that of si-NC $(p<0.05)$, while the ratio of firefly luciferase to Renilla luciferase in MG63 cells transfected with psiCHECKPLD1-WT plasmid and si-SP1 had no significant difference with si-NC control ( $p>0.05)$, indicating that the decrease of Firefly luciferase to Renilla luciferase ratio was due to the expression of reporter gene after SP1 binding to PLD1 promotor/enhancer. It was proved that SP1 could bind and target with PLD1 promotor/ enhancer region (fig. 2).

The cell viability of MG63 cells treated with TSA or not was measured by CCK-8 analysis. As shown in fig. 3, after TSA treatment for $24 \mathrm{~h}$, the proliferation rate of MG63 cells was $(65.20 \% \pm 2.31 \%)$, which was significantly lower than that of PBS control group $(96.63 \% \pm 1.14 \%)(\mathrm{p}<0.05)$.

Cell formation assay showed that the number of MG63 cells treated with TSA decreased significantly $(p<0.05)$, indicating that TSA could inhibit the proliferation of osteosarcoma cells. The photos and statistical results are shown in fig. 4.

TABLE 1: THE PRIME SEQUENCES FOR QRT-PCR

\begin{tabular}{lll}
\hline Gene & \multicolumn{1}{c}{ Forward primer sequence } & \multicolumn{1}{c}{ Reverse primer sequence } \\
\hline SP1 & 5' 'CACCAGAATAAGAAGGGAGG-3' & 5'-GGTGGTAATAAGGGCTGAA-3' \\
PLD1 & 5'-AATCGTTGGAGGTTGGACTG-3' & 5'-AGACGGTGGATGACACATGA-3' \\
B-actin & 5'-AGCGAGCATCCCCCAAAGTT-3' & 5'-GGGCACGAAGGCTCATCATT-3' \\
p21 & 5'-TGTCTTGTACCCTTGTGCCTCG-3' & 5'-AATCTGTCATGCTGGTCTGCC-3' \\
Survivin & 5'-TCCCTGGCTCCTCTACTG TT-3' & 5'-TGTCTCCTCATCCACCTGAA-3' \\
Cyclin A2 & 5'-TCCTCGTGGACTGGTTAGTTGA-3' & 5'-GCACTGACATGGAAGACAGGAA-3' \\
\hline
\end{tabular}
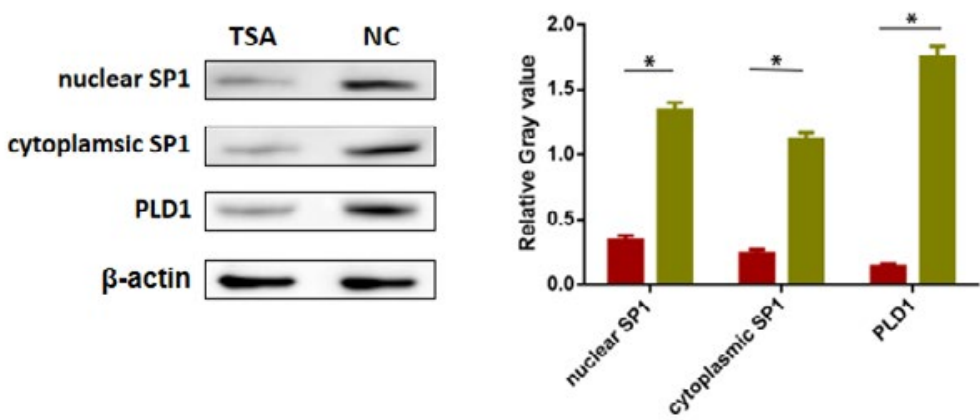

Fig. 1: Western blot of PLD1, nuclear and cytoplasmic SP1 ( $\square$ ): TSA ( $\square$ ) NC $(* \mathrm{p}<0.05$ vs NC, $\mathrm{n}=6)$ 


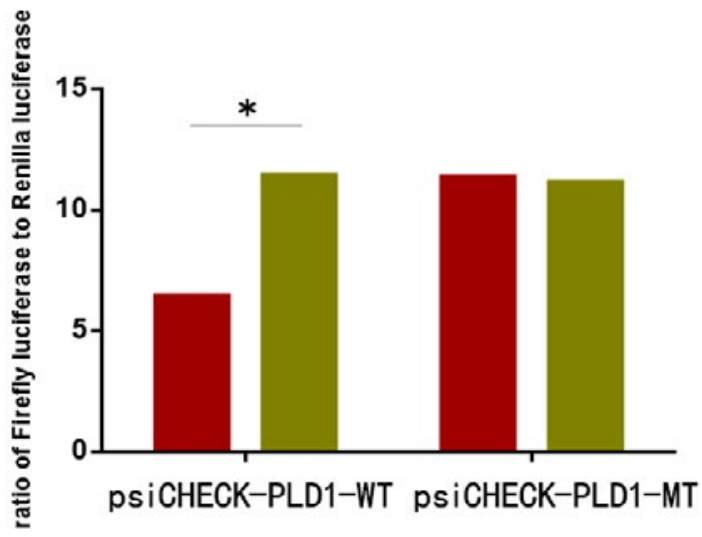

Fig. 2: Dual luciferase reporter gene assay result ( $\square$ ) si-SP1 ( ) si-NC

$\left({ }^{*} \mathrm{p}<0.05\right.$ vs si-NC)

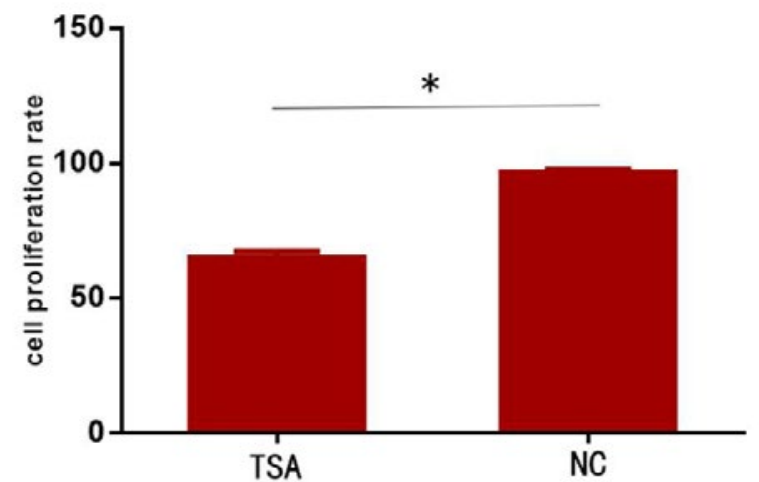

Fig. 3: CCK-8 assay result $\left({ }^{*} \mathrm{p}<0.05\right.$ vs NC, $\left.n=6\right)$
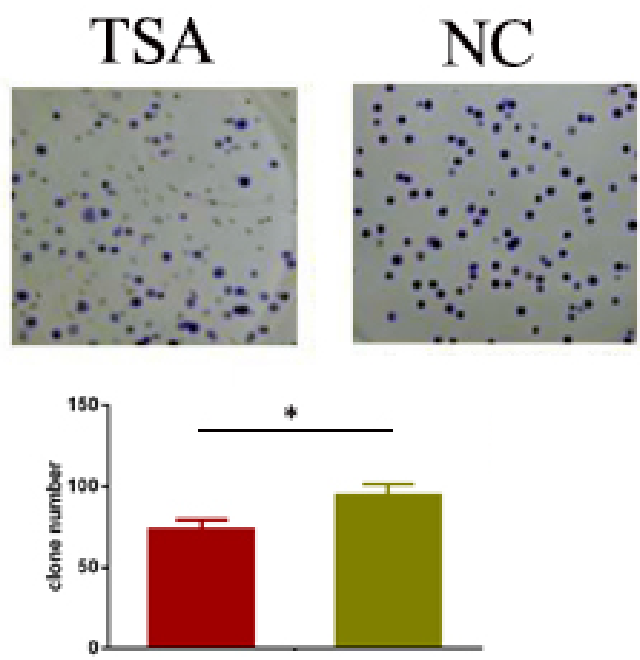

Fig. 4: Photos and result of clone formation assay ( ): TSA ( $) \mathrm{NC}$

$(* \mathrm{p}<0.05$ vs $\mathrm{NC}, \mathrm{n}=5)$

As shown in fig. 5, the gene expression of SP1 and PLD1 in TSA-treated MG63 cells decreased significantly compared with TSA free cells $(\mathrm{p}<0.05)$. The gene expression levels of survivin which inhibits cells apoptosis in TSA-treated MG63 cells were significantly lower than that in TSA free cells $(p<0.05)$. The gene expression levels of $\mathrm{p} 21$ which induces cells apoptosis in TSA-treated MG63 cells were significantly higher than that in TSA free cells $(p<0.05)$. The expression of cyclin A2 gene had no significant difference between two groups $(\mathrm{p}<0.05)$.

As shown in fig. 6, TSA can increase the early apoptosis rate,late apoptosis rate and total apoptosis rate of MG63 cells compared to the non-TSA-treatment cells $(\mathrm{p}<0.05)$ (fig. 5).

HDACi is a new class of antitumor drugs by blocking cell cycle, inducing apoptosis and differentiation. Histone deacetylation is harmful to DNA and histone for preventing dissociation of protein octamer, then inhibits the specific binding of various transcription factors and co transcription factors with DNA binding sites and

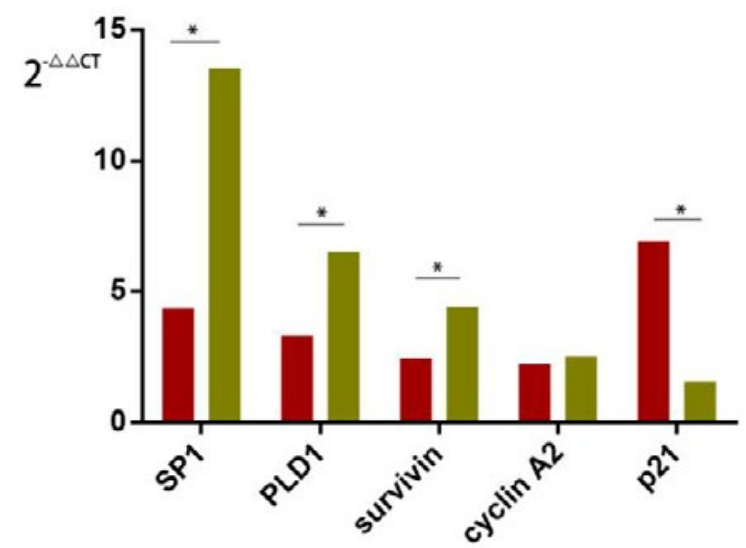

Fig. 5: PCR assay result ( $\square$ ): TSA ( $\square$ NC $(* \mathrm{p}<0.05$ vs NC)
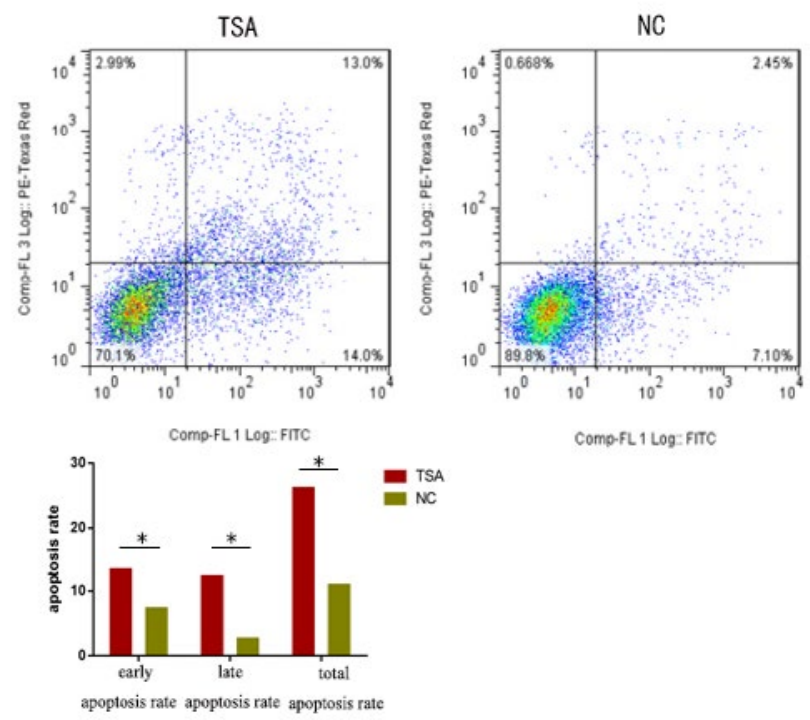

Fig. 6: Flow cytometry result for apoptosis rate ( $\square)$ : TSA ( $\mathrm{NC}$

$\left({ }^{*} \mathrm{p}<0.05\right.$ vs NC) 
gene transcription. For the treatment of osteosarcoma, HDAC inhibitors such as AR-42 and TSA can activate Akt, p53, VEGI / DR3 related pathways and promote osteosarcoma cell apoptosis. It has been reported that the expression of SP1 in bladder cancer cells was decreased by TSA. In recent years, several studies have suggested that transcription factor SP1 is a tumor promoter for colon cancer ${ }^{[10]}$ and prostate cancer $^{[11]}$. SP1 plays an important role in regulating tumor related gene expression by self-modifying protein complex structure and HDAC inhibitors ${ }^{[12]}$. Our previous studies have shown that SP1 inhibitor mithramycin A and transfection of SP1 siRNA to human osteosarcoma cell line MG63 could decrease the protein expression and promoter activity of PLD1, indicating that SP1 may participate in the pathogenesis and progress of osteosarcoma by regulating PLD1 expression. PLD1 present widely in different tissues and cells and are activated by a wide variety of receptor tyrosine kinases (RTKs) and G protein-coupled receptors (GPCRs) ${ }^{[13]}$. Biological function of PLD1 includes regulated exocytosis, endocytosis, proliferation,cell migration, Golgi-ER trafficking, autophagy, and apoptosis, which encompasses almost all signaling-driven processes ${ }^{[14]}$. Interest has become increasingly focused on PLD1 in thecontext of cancer. Therefore, based on the literatures and our previous research results, we speculate that HDACi may target and regulate SP1 and effect the expression of PLD1, thus inhibit the proliferation and promote cells apoptosis of osteosarcoma cells.

In this article, we used TSA, a typical and extensive HDACi, to treat osteosarcoma cell line MG63 in vitro. qPCR and Western blot results showed that after TSA treatment, SP1 in nucleus and cytoplasm of MG63 both decreased significantly, and PLD1 also decreased significantly. These results indicated that HDACi can inhibit the expression of SP1 protein and nuclear migration, thus inhibiting the expression of PLD1. In addition, this study demonstrated that SP1 could bind to the promoter / enhancer fragment of PLD1 gene by dual luciferase reporter gene assay. So SP1 is the regulatory transcription factor of PLD1 in osteosarcoma cells. TSA inhibits the expression of PLD1 by inhibiting SP1. Then, CCK-8 test and clone formulation analysis showed that TSA could reduce the proliferation level and cell activity of MG63 cells. The results of qPCR showed that TSA could inhibit survivin and increase p $21^{[15]}$. The results of flow cytometry showed that TSA could promote the early and late apoptosis rates of MG63 cells.
In conclusion, SP1 could bind and target with PLD1 promotor/enhancer region. HDACi can reduce the proliferation and apoptosis of osteosarcoma cells through decreasing the expression of PLD1 by inhibiting the protein expression and nuclear migration of SP1.

\section{Authors' contributions:}

Jian qin conceived and designed the experiments; Tangbo Yuan and Zhen Jin performed the experiments; Dawei Cai analyzed the data and wrote the paper.

\section{Acknowledgements:}

This work was supported by the Natural Science Foundation of the Jiangsu Higher Education Institutions of China (Grant No. 18KJB320009).

\section{Conflicts of interest:}

The authors report no conflicts of interest.

\section{REFERENCES}

1. Baldini N, Scotlandi K, Barbanti-brò D. Expression of P-glycoprotein in high-grade osteosarcomas in relation to clinical outcome. N Engl J Med 1995;333:1380-5.

2. Wadayama B, Toguchida J, Shimizu T. Mutation spectrum of the retinoblastoma gene in osteosarcomas. Cancer Res 1994;54:3042-8.

3. Tennis MA, Vanscoyk M, Keith RL. The role of prostacylin in lung cancer. Transl Res 2010;155:57-61.

4. Kang DW, Choi KY. Phospholipase D means Wnt signaling: a new target for cancer therapy. Cancer Res 2011;71:293-7.

5. Bae IH, Park MJ, Yoon SH. Bcl-w promotes gastric cancer cell invasion by inducing matrix metalloproteinase-2 expression via phosphoinositide 3-kinase, Akt, and SP1. Cancer Res 2006;66:4991-5.

6. Xu X, Wang Y. Tissue microarray staging reveals PLD1 and SP1 have a collaborative, pro tubular effect in patients with osteosarcomas. Oncotarget 2017;8:74340-7.

7. Khan O, La Thangue NB. HDAC inhibitors in cancer biology: emerging mechanisms and clinical applications. Immunol Cell Biol 2012;90:85-94.

8. Wagner JM, Hackanson B, Lübbert M. Histone deacetylase (HDAC) inhibitors in recent clinical trials for cancer therapy. Clin Epigenetics 2010;1:117.

9. Zhao XY, Xing FJ, Cong YW. Antimony trichloride induces a loss of cell viability via reactive oxygen species-dependent autophagy in A549 cells. Int J Biochem Cell Biol 2017;93:3240.

10. Takami Y, Russell MB, Gao C. SP1 regulations osteopontin expression in SW480 human Colon adenocarcinoma cells. Surgery 2007;142:163-9.

11. Sroka IC, Nagle RB, Bowden GT. Membrane-type 1 matrix metal loproteinase is regulated by SP1 through the Differential Activation of AKT, JNK, and ERK Pathways in Human Prostate Tumor cells. Neoplasia 2007;9:406-17.

12. Capobianco E, Mora A, LaSala D. Separate and combined effects of DNMT and HDAC inhibitors in treating human multi-drug resistant osteosarcoma HosDXR150 cell line. PloS one 2014;9:95596. 
13. Vadovič P, Šamajová O, Takáč T. Biochemical and Genetic Interactions of Phospholipase D Alpha 1 and MitogenActivated Protein Kinase 3 Affect Arabidopsis Stress Response. Front Plant Sci 2019;10:275.

14. Hassan BM, Adnan KM, Mabood HF. Interfering with PLD1-
PED/PEA15 interaction via self-inhibitory peptides: an in silico study to discover novel therapeutic candidates against type 2 diabetes. Saudi Journal of Biological Sciences 2018;26.

15. Ren ZP, Hu YC, Li GS. The expression of HIF-1 $\alpha$ induced long noncoding RNA FOXD2-AS1 promotes the osteosarcoma through expressing p21. Biomed Pharmacol 2019;117:109104. 